\title{
ABORDAGEM TERAPÊUTICA DOS DISTÚRBIOS RESPIRATÓRIOS DO SONO TRATAMENTO COM VENTILAÇÃO NÃO-INVASIVA (CPAP, BiPAP E AUTO-CPAP)
}

\author{
THERAPEUTIC APPROACH OF RESPIRATORY SLEEP DISORDERS \\ TREATMENT WITH NON-INVASIVE VENTILATION (CPAP, BIPAPAND AUTO-CPAP)
}

Geruza A Silva', Daniela V Pachito²

\begin{abstract}
${ }^{1}$ Docente. Disciplina de Pneumologia. Departamento de Clínica Médica. Faculdade de Medicina de Ribeirão Preto-USP. ${ }^{2}$ Médica Adido. Setor de Neurofisiologia Clínica. Hospital das Clínicas da Faculdade de Medicina de Ribeirão Preto-USP

Correspondência: Profa. Dra. Geruza Alves da Silva - E-mail: gadsilva@fmrp.usp.br

Hospital das Clínicas da Faculdade de Medicina de Ribeirão Preto-USP. Departamento de Clínica Médica.

Av. dos Bandeirantes, 3900. Bairro Monte Alegre. CEP: 14048-900 - Ribeirão Preto-SP
\end{abstract}

Silva GA, Pachito DV. Abordagem terapêutica dos distúrbios respiratórios do sono. Tratamento com ventilação não-invasiva (CPAP, BiPAP e Auto-CPAP). Medicina (Ribeirão Preto) 2006; 39 (2): 212-217.

RESUMO: A abordagem dos distúrbios respiratórios do sono com uso de pressão positiva contínua nas vias aéreas é considerada a forma mais eficiente de tratamento. É feita por meio de aparelho apropriado, chamado CPAP que se adapta a um tubo flexível através do qual o ar liberado pelo aparelho é conduzido até uma máscara firmemente adaptada ao nariz do paciente. Os portadores de distúrbios graves bem como os moderados sintomáticos, aderem facilmente a essa forma de tratamento. A adesão ao tratamento pode ser melhorada com medidas simples em alguns casos. As complicações são previsíveis e raras. Outras modalidades de dispositivos de pressão positiva ocasionalmente são mais bem toleradas e mais eficazes para o tratamento de hipoventilação alveolar e apnéias centrais.

Descritores: Pressão Positiva Contínua nas Vias Aéreas. Síndromes da Apnéia do Sono. Ronco.

\section{1- INTRODUÇÃO}

A forma mais conservadora de tratamento das apnéias do sono e da hipoventilação da obesidade é também a mais bem aceita pelos pacientes. É ainda, considerada a forma mais eficiente de tratamento. Consiste do uso de pressão positiva aplicada as vias aéreas superiores durante o sono, através de máscara nasal ou facial.

Atualmente existem diferentes modos de aplicação da pressão positiva nas vias aéreas: a) o modo clássico aplicado à maioria dos pacientes, utiliza pres- são positiva contínua por meio de dispositivo apropriado chamado aparelho de CPAP (Continuous Positive Airway Pressure); b) outro modo, geralmente aplicado aos pacientes obesos hipercapneicos, utiliza pressão positiva em dois níveis, inspiratório e expiratório, por meio de aparelho de BiPAP (Bi-level Positive Airway Pressure); c) por fim, aparelho com ajuste automático dos níveis de pressão positiva denominado de Auto-CPAP constitui uma variante do método clássico ficando reservado a situações mais específicas. 


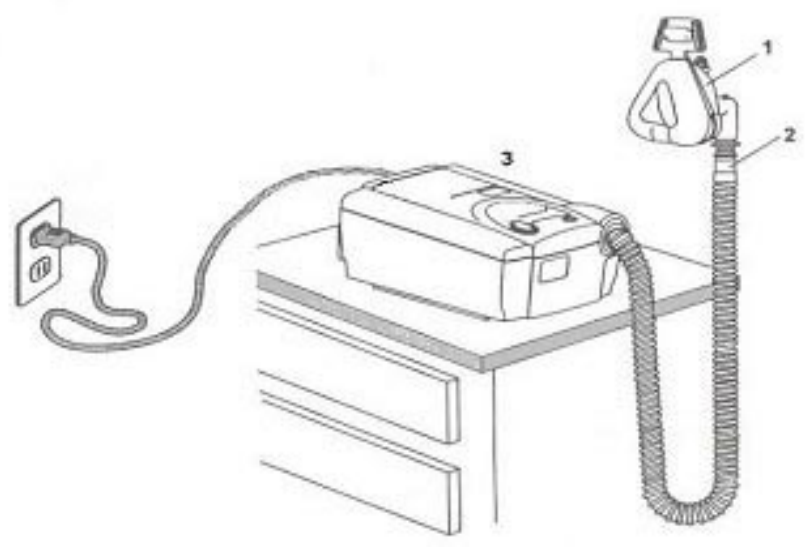

Figura 1: Representação esquemática do aparelho de CPAP. O aparelho funciona com a acoplagem de acessórios indispensáveis ao funcionamento do sistema, a saber: 1) máscara nasal (ou facial, em casos especiais); 2) tubo flexível para conexão da máscara ao aparelho; 3 ) aparelho de CPAP.

\section{2- O QUE É O CPAP E COMO AGE SOBRE AS VIAS AÉREAS?}

O aparelho de CPAP foi primariamente concebido para tratamento dos distúrbios respiratórios do sono, pelo pneumologista australiano C. E. Sullivan no início da década de $1980^{1}$. Atua dentro de uma escali de pressão que vai de 0 a $20 \mathrm{cmH}_{2} \mathrm{O}$. Ao longo dos anos subseqüentes, sua eficácia vem sendo observa. da no auxílio a resolução de diversos outros problemas respiratórios em adultos e crianças. Aqui, nos restringiremos à descrição do uso do aparelho nas apnéias obstrutivas do sono.

O CPAP possui um mecanismo intrínseco que lhe permite aspirar ar do meio ambiente, filtrá-lo e enviá-lo ao paciente através de tubo flexível (Figura 1). $\mathrm{O}$ ar penetra nas vias aéreas, através de máscara nasal, sob pressão fixa, pré-estabelecida para cada paciente. A pressão eficaz situa-se geralmente na faixa de 5 a 13 $\mathrm{cmH}_{2} \mathrm{O}$. O ar sob pressão penetrando nas vias aéreas impede o colapso das paredes musculares faringeanas, evitando a ocorrência das apnéias, hipopnéias e de respiração com esforço aumentado produzindo despertares. $\mathrm{O}$ aparelho impede também a vibração de outras estruturas moles da faringe, evitando o ronco.

$\mathrm{O}$ efeito do CPAP sobre as vias aéreas superiores pode ser representado pelo clássico esquema na Figura 2.

\section{3- COMO ESTABELECER A PRESSÃO IDEAL A SER EMPREGADA NO CPAP?}

O paciente com distúrbio respiratório do sono, candidato ao uso de CPAP é submetido a uma segunda polissonografia, acrescentando-se o aparelho ao circuito de registro das mesmas variáveis utilizados para obtenção do diagnóstico (ver capítulo II sobre monitorização do paciente para polissonografia).

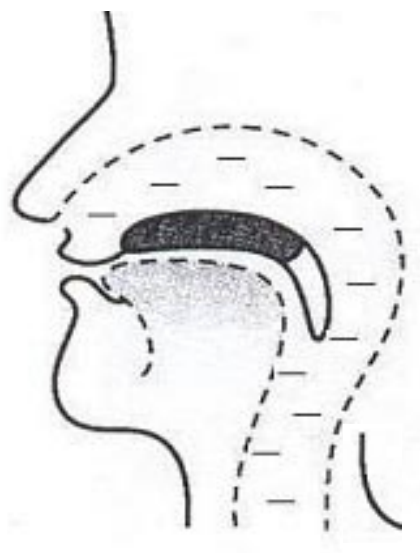

A

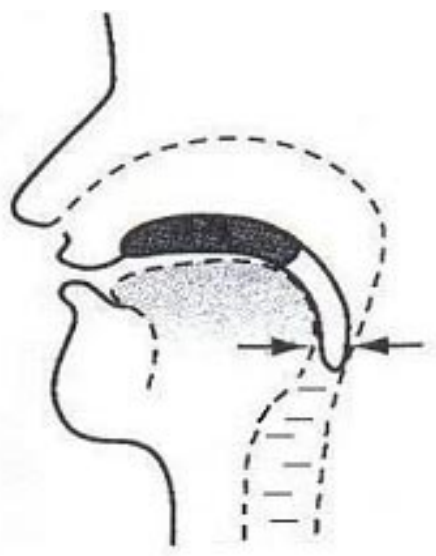

B

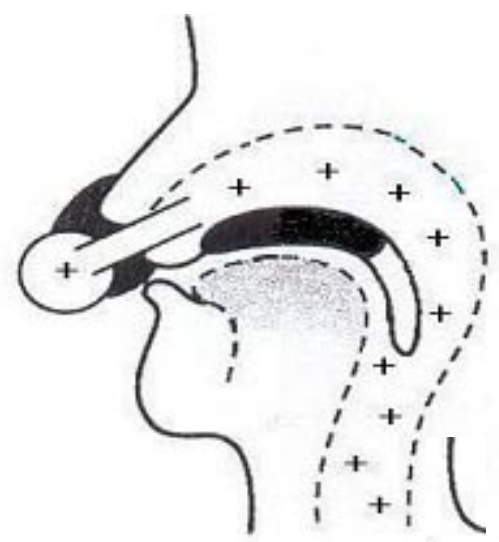

C

Figura 2: A figura $2 \mathrm{~A}$ representa a distribuição da pressão negativa que se desenvolve nas vias aéreas no início da inspiração espontânea. Nos distúrbios respiratórios do sono, a resistência anormalmente alta das vias aéreas superiores (ver capítulo III sobre apnéias obstrutivas do sono) retarda a passagem do ar através destas vias, retardando a anulação da pressão negativa. A persistência momentânea dessa condição promove o colapso das vias aéreas em um ou mais pontos da sua porção susceptível, o canal faringeano (naso, oro e hipofaringe), figura 2B.

A figura $2 \mathrm{C}$ ilustra o efeito da aplicação de pressão positiva nas vias aéreas por meio do aparelho de CPAP. A restauração das condições de pressão positiva e manutenção desse estado, assegura a permeabilidade das vias aéreas (adaptado de Grunstein R. Continuous positive airway pressure treatment for obstructive sleep apnea-hypopnea syndrome 2005$)^{2}$. 
O ajuste é feito no sentido ascendente e descendente com o objetivo de vencer o fenômeno de "hysteresis" que existe na relação entre CPAP e resistência das vias aéreas. Esse procedimento permite o ajuste da pressão em níveis mais baixos que quando limitado a uma busca apenas ascendente, de obtenção da pressão ideal.

O tempo necessário para o acerto da pressão é variável. Idealmente, a pressão é determinada durante estudo de noite inteira, com o tempo total de sono superior a 180 minutos. Pacientes com quadros graves e sonolência diurna marcante podem ser submetidos ao protocolo chamado "split-night", no qual a primeira metade da noite é destinada ao diagnóstico, enquanto a titulação da pressão é realizada na segunda metade $^{3}$. Em nosso laboratório, freqüentemente são realizados ajustes durante o sono diurno por 3-5 horas, com bons resultados; essa conduta não é amplamente praticada, mas encontra suporte ${ }^{4}$.

O ajuste da pressão busca abolir todos os eventos respiratórios anormais (apnéias, hipopnéias, despertares por esforço respiratório aumentado, limitações ao fluxo aéreo, roncos e dessaturação da hemoglobina). Durante a titulação deve ocorrer registro de sono REM, pois nessa fase predominam os eventos respiratórios obstrutivos, estando o paciente em decúbito dorsal, posição facilitadora dos distúrbios ${ }^{5,6}$.

Os pacientes que manifestam persistência de sintomas como, sono não restaurador e sonolência diurna excessiva, apesar do uso correto do aparelho, devem ser reavaliados com estudo polissonográfico.

\section{4- ADESÃO AO CPAP}

A adaptação do paciente ao aparelho de CPAP constitui, na maioria das vezes, sua única possibilidade de tratamento não-invasivo (não-cirúrgico). O uso de CPAP é considerado o tratamento "padrão-ouro" para apnéia do sono por vários aspectos entre eles, o de ser não-invasivo. Em média os usuários utilizam o aparelho por 4,5 a 5 horas/noite ${ }^{7}$. Em cada lado da faixa de adesão ao tratamento estão os usuários regulares e os irregulares sendo que, os primeiros constituem $60 \%$ do total e consistem de pacientes que usam o aparelho quase todas as noites por 6 horas ou mais; os demais são usuários irregulares com todo tipo de variação no tempo de uso por semana. Adesão ao CPAP significa usá-lo por 6 horas/noite, 6-7 dias/semana ${ }^{5}$.

Os pacientes com distúrbios graves são os que melhor aderem ao tratamento. Neles, o tratamento com CPAP produz verdadeira e benéfica mudança de vida, graças à considerável melhora dos sintomas relacionados a hipersonolência diurna. Isso favorece uma motivação para o uso regular do aparelho, durante todo o período de sono de todos os dias da semana. Infelizmente, essa ótima adesão não ocorre em todos os pacientes graves. Por alguma razão, alguns poucos pacientes graves, independentemente do índice de massa corpórea, aderem só parcialmente, ou mesmo, não aderem ao tratamento com $\mathrm{CPAP}$, necessitando tentativas com outras modalidades de tratamento que incluem o BiPAP (ver adiante).

A disposição do paciente, para aceitar o aparelho, reduz muito os casos de falha do tratamento e nesse sentido a correta orientação ao paciente quanto às dificuldades e benefícios inerentes ao tratamento é fundamental.

Grande parte dos pacientes com apnéias do sono de grau moderado, e pequena parte daqueles com distúrbio leve, desde que sintomáticos, também apresentam boa adesão. Os sintomas mais capazes de promover disposição para aderir ao tratamento são: hipersonolência diurna, ou a presença de doença cardiovascular conscientizando o paciente da necessidade do tratamento.

A baixa adesão é nitidamente observada nas seguintes circunstâncias: a) em pacientes jovens; b) em pacientes solteiros com sintomas leves ou moderados; c) nos distúrbios respiratórios do sono, de grau leve; d) nos pacientes com doença pulmonar obstrutiva crônica, subjacente.

\section{5- ATITUDES RECOMENDADAS PARA FA- CILITAR A ADESÃO}

É possível promover ou melhorar a aceitação do CPAP por meio das seguintes providências:

1) identificando e corrigindo fatores mecânicos removíveis, localizados no nariz ou na faringe;

2) evitando o ressecamento das vias aéreas por meio de umidificação do ar inspirado e garantido o fechamento da boca durante o sono;

3) promovendo o tratamento de infecções dos seios da face;

4) corrigindo falhas de ajuste da máscara ao rosto, de modo a evitar vazamentos de ar nos olhos, reação cutânea traumática, deslocamento da máscara com os movimentos corporais no sono;

5) reavaliando a pressão nos casos de dificuldade de expiração;

6) disponibilizando acesso a consultas especializadas durante as primeiras semanas de tratamento para identificar e corrigir dificuldades de adesão. 


\section{6- COMPLICAÇÕES DO USO DE CPAP}

As complicações decorrentes do uso de CPAP são previsíveis e raras. Como em todo procedimento aplicado ao ser humano com finalidade diagnóstica ou terapêutica, o emprego do CPAP necessita contar com o bom senso e responsabilidade médicas. $\mathrm{O}$ acesso ao controle da pressão do aparelho é vetado ao paciente, ficando a escolha da pressão, sob total responsabilidade do médico; o profissional paramédico (ou vendedor) que repassa o aparelho calibrado para o paciente, obedece à prescrição médica. É importante lembrar que a pressão a ser introduzida nas vias aéreas pode produzir danos num parênquima pulmonar enfisematoso, ou tomar caminho através dos seios da face uma vez que estas estruturas são formações saculares em contigüidade com as fossas nasais e que estão situadas na caixa craniana. As complicações descritas são:

- Desconforto torácico;

- Piora da congestão nasal e da rinorréia, em pacientes com rinite alérgica.

- Pneumotórax;

- Pneumoencéfalo;

- Dor em seios da face;

- Sinusopatia;

- Ressecamento nasal;

- Enfisema subcutâneo nas pálpebras inferiores;

- Aerofagia;

- Epistaxe.

Alguns problemas relacionados ao uso de CPAP são de difícil solução, como:

a) sono interrompido por ruído proveniente do aparelho com alta pressão e b) intolerância do cônjuge.

\section{7- QUAIS OS RESULTADOS ESPERADOS DO TRATAMENTO COM CPAP?}

Levando-se em conta que o papel do CPAP é reverter a oclusão e manter a permeabilidade das vias aéreas no sono, fica fácil perceber a extensão do benefício proporcionado à saúde dos usuários dessa forma de tratamento. O benefício mais imediato ocorre sobre o estado de sonolência diurna. Este é um sintoma comum e debilitante que está presente na maioria dos casos triados para investigação de distúrbio respiratório do sono. Mas o benefício decorrente da supressão das apnéias do sono que se obtém com o CPAP, vai alem da restauração da continuidade, da quantidade e da distribuição dos estágios do sono: é garantia de oxigenação normal para os tecidos e órgãos e normalização da produção de catecolaminas. Esses fatores asseguram no mínimo, a parada da progressão de diversos outros mecanismos de ordem neural, humoral, metabólico, trombótico e inflamatório de produção de doenças, incluindo a hipertensão arterial sistêmica que se desenvolve em $30 \%$ dos pacientes com síndrome das apnéias obstrutivas do sono ${ }^{8,9}$.

\section{8- FORMAS VARIANTES DE APLICAÇÃO DE PRESSÃO POSITIVA NAS VIAS AÉREAS BiPAP E AUTO-CPAP}

Outros dispositivos de pressão positiva têm sido desenvolvidos com o intuito de melhorar a tolerância e a adesão ao tratamento, e permitir o controle de outras condições como hipoventilação alveolar e apnéias centrais.

\section{A) Bi-level Positive Airway Pressure (BiPAP)}

A pressão necessária para manter a patência das vias aéreas durante a expiração é menor em relação à inspiração. $\mathrm{O}$ aparelho de BiPAP permite a oferta de dois níveis pressóricos distintos durante a inspiração e a expiração. A redução da pressão expiratória diminui o trabalho respiratório relacionado à expiração em vigência de pressão positiva, tornando o método mais confortável. A possibilidade de ajuste individualizado permite a utilização de pressão inspiratória em níveis que não seriam tolerados de forma contínua. Além disso, a diferença pressórica facilita o aumento da ventilação, benéfica nos casos de hipoventilação alveolar.

A maioria dos aparelhos possibilita o funcionamento em modo assistido e/ou controlado, sendo ainda controverso qual seria o mais efetivo. Em pacientes com apnéias centrais prolongadas, o modo controlado é freqüentemente utilizado, para garantir a ventilação adequada.

As principais indicações de utilização de BiPAP são: presença de apnéias centrais clinicamente significativas, hipoventilação alveolar primária ou secundária e presença de $\mathrm{DPOC}^{10}$, doenças restritivas pulmonares ou extrpulmonares (neuromuscular ou do arcabouço ósseo). Adicionalmente, pacientes com dificuldade de adaptação ao aparelho de CPAP tendem a tolerar melhor o BiPAP.

Cerca de 8.9 a $48 \%$ dos pacientes portadores de distúrbio respiratório do sono apresentam controle 
insatisfatório de apnéias e hipopnéias, ou dessaturação relacionada a hipoventilação apesar da utilização de CPAP $^{11,12,13}$. Falta de resposta ao CPAP é previsível nas seguintes situações: obesidade mórbida, em face da queixa de dispnéia, alta percentagem de tempo total de sono com saturação abaixo de $90 \%$, alteração gasométrica durante a vigília e aumento da $\mathrm{PaCO} 2$ após exercício físico ${ }^{13}$. Pacientes com tais características podem se beneficiar do tratamento com o BiPAP.

\section{B) Auto-CPAP}

Os aparelhos de Auto-CPAP possuem sistema de detecção de eventos respiratórios anormais e geração automática da pressão positiva ofertada, de acordo com a necessidade momentânea. Esta necessidade é variável entre noites diferentes e mesmo ao longo da mesma noite. Pacientes portadores de síndrome de apnéia/hipopnéia obstrutiva do sono freqüentemente necessitam de maior pressão quando em decúbito dorsal, pelo deslocamento de estruturas moles que compõem a faringe (palato mole, língua, úvula) e durante o sono REM, devido à atonia muscular típica desta fase de sono. Além disso, a colapsibilidade da faringe pode ser influenciada por vários fatores, como o uso de bebidas alcoólicas ou de substâncias miorelaxantes, presença de quadros infecciosos e de rinite alérgica, variação do peso corpóreo. A variação pressórica dos aparelhos de Auto-CPAP pode levar a uma redução da pressão média ofertada ao longo da noite, proporcionando, teoricamente, maior conforto e facilidade de adesão ao tratamento. Ainda não existem dados que assegurem a existência de maior adesão a esses aparelhos em relação ao CPAP convencional.
De acordo com os parâmetros práticos da Academia Americana de Medicina do Sono, os aparelhos de AutoCPAP devem ser utilizados somente após diagnóstico de SAHOS realizado através de polissonografia, excluindo-se as seguintes condições: insuficiência cardíaca congestiva, doenças pulmonares como DPOC e hipoventilação relacionada à obesidade ${ }^{14}$

Os algoritmos utilizados para a detecção dos eventos respiratórios obstrutivos diferem entre os vários tipos de aparelhos de Auto-CPAP. Alguns estudos sugerem que dispositivos com algoritmo de limitação de fluxo medido por pneumotacógrafo controlam melhor os eventos obstrutivos do que aqueles que apresentam transdutor de pressão para detecção de vibração ${ }^{15,16}$. Estes são contra-indicados para pacientes previamente submetidos à uvulopalatofaringoplastia os quais podem ter apnéias porem não apresentam ron$\cos ^{16}$. A superioridade de uma tecnologia em relação a outra não está estabelecida.

A pressão deve ser ajustada para o aparelho funcionar dentro de uma faixa de variação.Alguns autores determinam uma pressão fixa por titulação com aparelho de CPAP convencional, ajustando em seguida a pressão do Auto-CPAP para uma faixa entre 4 pontos abaixo e 2 pontos acima, daquela titulada no CPAP convencional. É possível ainda utilizar toda a faixa de atuação do aparelho, ou seja, de 3 até 20 $\mathrm{cmH}_{2} \mathrm{O}$.

É necessário observar que pacientes sob essa modalidade de tratamento devem ser criteriosamente acompanhados e a persistência ou o reaparecimento de sintomas sugestivos de SAHOS indica a necessidade de reavaliação com polissonografia.

Silva GA, Pachito DV. Therapeutic approach of respiratory sleep disorders . Treatment with non-invasive ventilation (CPAP, BiPAP and Auto-CPAP). Medicina (Ribeirão Preto) 2006; 39 (2): 212-217.

ABSTRACT: Continuous positive airway pressure has proved to be the most efficient way of treatment for respiratory sleep disorders. The device called CPAP is connected to a patient by means of a flexible tube conducting the delivered air up to a mask tightly attached to the nose of the patient. The patients with severe or symptomatic moderate sleep respiratory disturbance easily adhere to this form of treatment. This adhesion can be improved with simple attitudes in some cases. The complications are predictable and uncommon. Some other kinds of positive pressure can be better tolerate and more efficient in the treatment of primary or secondary alveolar hypoventilation and central apneas.

Keywords: Continuous Positive Airway Pressure. Sleep Apnea Syndromes. Snoring. 


\section{REFERÊNCIAS}

1 - Sullivan CE, Berthon-Jones M, Issa FG, Eves L. Reversal of obstructive sleep apnoea by continuous positive airway pressure applied through the nares. Lancet 1981; 1:862-5.

2 - Grunstein R. Continuous positive airway pressure treatment for obstructive sleep apnea-hypopnea syndrome. In: Krieger $\mathrm{MH}$, Roth T, Dement WC, eds. Principles and practice of sleep medicine. $4^{\text {th }}$ ed. Philadelphia: Elsevier Saunders; 2005. p.106680 .

3 - Standards of Practice Committee of the American Academy of Sleep Medicine. Practice parameters for the indications for polisomnography and related procedures. Polisomnography Task Force, American Sleep Disorders Association Standards of Practice Committee. Sleep 1997; 20:406-22.

4 - Rosenthal L, Nykamp K, Guido P, Syron ML, Day R, Rice M, Roth T. Daytime CPAP titration: a viable alternative for patients with severe obstructive sleep apnea. Chest 1998;114:1056-60.

5 - Grunstein RR. Sleep-related breathing disorders: 5 nasal continuous positive airway pressure treatment for obstructive sleep apnoea. Thorax 1995;50:1106-13.

6 - Montserrat JM, Ballester E, Olivi H, Reolid A, Lloberes P, Morello A, Rodriguez-Roisin R. Time-course of stepwise CPAP titration: behavior of respiratory and neurological variables. Am J Respir Crit Care Med 1995;152:1854-9.

7 - Kribbs NB, Pack Al, Kline LR, Smith PL, Schwartz AR, Schubert NM, Redline S, Henry JN, Getsy JE, Dinges DF. Objective measurement of pattern of nasal CPAP use by patients with obstructive sleep apnea. Am Rev Respir Dis 1993;147: 887-95.

8 - Vgontzas AN, Bixier EO, Chrousos GP. Metabolic disturbances in obesity versus sleep apnoea: the importance of visceral obesity and insulin resistance. J Intern Med 2003;254:32-44.
9 - Heitmann J, Ehlenz K, Penzel T, Becker HF, Grote L, Voigt $\mathrm{KH}$, Hermann Peter J, Vogelmeier C. Sympathetic activity is reduced by nCPAP in hypertensive obstructive sleep apnoea patients. Eur Respir J 2004;23:255-62.

10 - Resta O, Guido P, Picca V, Sabato R, Rizzi M, Scarpelli F, Sergi M. Prescription of nCPAP and nBiPAP in obstructive sleep apnoea syndrome: Italian expierence in 105 subjects. A prospective two centre study. Resp Med 1998; 92: 820-7.

11 - Becker H, Brandenburg U, Peter JH, Schneider H, Weber K, von Wichert P. Indications and application of BiPAP therapy. Pneumologie 1993;47 (Suppl 1): 184-8.

12 - Sanders MH, Kern NB, Stiller RA, Strollo Jr PJ, Martin TJ, Atwood Jr CW. CPAP therapy via oronasal mask for obstructive sleep apnea. Chest 1994; 106: 774-9.

13 - Schäfer H, Ewig S, Hasper E, Lüderitz B. Failure of CPAP therapy in obstructive sleep apnoea syndrome: predictive factors and treatment with bilevel-positive airway pressure. Resp Med 1998; 92: 208-15.

14 - Littner M, Hirshkowitz M, Davila D, Anderson Wm, Kushida CA, Woodson BT, Johnson SF, Merrill SW, Standards of Practice Committee of the American Academy of Sleep Medicine. Practice parameters for the use of auto-titrating continuous positive airway pressure devices for titrating pressures and treating adult patients with obstructive sleep apnea syndrome. An American Academy of Sleep Medicine Report. Sleep 2002, 25;143-7.

15 - Shi HB, Cheng L, Nakayama M, Kakazu, Y, Yin M, Miyoshi A Komune S. Effective comparison of two auto-CPAP devices for treatment of sleep apnea based on polysomnographic evaluation. Auris Nasus Larynx 2005; 32: 237-41.

16 - Farre R, Montserrat JM, Rigau J, trepat X, Pinto P, Navajas D. Response of automatic continuous positive airway pressure devices to different sleep breathing patterns: a bench study. Am J Respir Crit Care Med 2002; 166: 469-73. 